\title{
Socioeconomic Status and Health-Related Quality of Life of Patients with Chronic Obstructive Pulmonary Disease
}

\author{
Marc Miravitlles $^{a} \quad$ Karlos Naberan $^{d} \quad$ Jordi Cantoni ${ }^{b} \quad$ Angel Azpeitia $^{c}$ \\ ${ }^{a}$ Fundació Clínic, Institut d'Investigacions Biomèdiques August Pi i Sunyer, Ciber de Enfermedades Respiratorias, \\ Hospital Clínic, ${ }^{b}$ Bioclever, and ${ }^{\mathrm{C}}$ Medical Department, Laboratorios Dr. Esteve S.A., Barcelona, and ${ }^{\mathrm{d}}$ Primary Health \\ Care Center Fuentes del Ebro, Zaragoza, Spain
}

For editorial comment see p. 400

\section{Key Words}

Chronic obstructive pulmonary disease - Educational level • Socioeconomic status $\cdot$ Health-related quality of life

\begin{abstract}
Background: Socioeconomic status (SES) is an important determinant of health and premature death. However, the impact of poor SES on the health status of patients with chronic obstructive pulmonary disease (COPD) has not been well determined. Objective: It was our aim to assess the impact of SES on the quality of life in COPD patients. Methods: This was a cross-sectional, observational, multicenter study. A total of 4,574 patients completed the EuroQol 5-dimension questionnaire (EQ-5D) and the Airways Questionnaire 20 (AQ20). SES was based on the subject's occupation and educational level. Occupational categories were based on the major group classification of the International Standard Classification of Occupations. Results: The mean age of the population was 67.1 years and the mean forced expiratory volume was $43.4 \%$. There was a gradient of impairment in health-related quality of life (HRQoL) according to the educational level, with significantly worse scores for the EQ-5D and the AQ20 for medium and low educational levels compared with high education $(p<0.001)$. Similarly, HRQoL was
\end{abstract}

also significantly impaired in more unskilled workers, with a gradient of the AQ20 from 8.6 units (SD 4.8) in class I to 10.1 units (4.6) in class $V(p<0.001)$ and from 0.75 units in class I to 0.63 units in class $V$ for the EQ-5D index, as well as from 62.9 units in class I to 55.6 units in class V for the EQ-5D visual analogue scale ( $p<0.001$ for all comparisons). These differences remained significant after controlling for covariates. Conclusions: Patients with a lower educational level and belonging to the unskilled professional groups had a poorer HRQoL. This is evident even in a country where access to health care services is universal and free.

Copyright $\odot 2011$ S. Karger AG, Basel

\section{Introduction}

Socioeconomic status (SES), whether measured by education, income or other indices of social class, has long been known to be associated with mortality from different diseases [1]. In respiratory health, the impact of socioeconomic disadvantage has been extensively studied in asthma, with the European Community Respiratory Health Survey I finding an increased prevalence of asthma in low SES groups [2]. It has also been demonstrated that individuals with low SES have a significantly in-

\section{KARGER}

Fax +4161306 1234

E-Mail karger@karger.ch

www.karger.com
(C) 2011 S. Karger AG, Basel

0025-7931/11/0825-0402\$38.00/0

Accessible online at:

www.karger.com/res
Marc Miravitlles

Servei de Pneumologia, Hospital Clínic

Villarroel 170

ES-08036 Barcelona (Spain)

Tel. +34 93227 5549, E-Mail marcm@ @epar.es 
creased risk of chronic bronchitis, respiratory symptoms and low lung function, independently of smoking $[1,3-5]$. The impact of low SES on respiratory disease in general has been attributed to poorer housing, more hazardous occupational exposure, poorer diet, a higher prevalence of smoking and even a higher frequency of second-hand smoking and respiratory infections in childhood $[1,6]$.

Previous population-based, epidemiological studies have observed that lower education was significantly and independently associated with a higher prevalence of chronic obstructive pulmonary disease (COPD) [7] and a higher probability of not being diagnosed early [8]. However, it is not clear if among patients with established COPD, the gradient of educational level and occupation has any impact on the disease in terms of a more severe impairment in health-related quality of life (HRQoL) even among patients with a similar degree of physiological deterioration.

We have conducted a nationwide survey among a large population of patients with COPD recruited from primary health care centers and respiratory specialists to ascertain the impact of SES on HRQoL and to examine this relationship after controlling for other variables such as age and severity of the baseline disease.

\section{Methods}

\section{Study Design}

The INSEPOC study (impacto del nivel socioeconómico en la calidad de vida de los pacientes con EPOC) was a cross-sectional, observational, multicenter study undertaken with the aim to evaluate the impact of the socioeconomic level in HRQoL of patients with COPD. To obtain maximal representativeness in terms of socioeconomic level and severity distribution of patients with COPD, both general practitioners and respiratory specialists were invited to participate. They were randomly selected from the database of the sponsoring company, which includes approximately $80 \%$ of the practicing general practitioners and $90 \%$ of the respiratory specialists registered in the country. Participating investigators were requested to include the first 3 consecutive unselected COPD patients who fulfilled the inclusion and exclusion criteria of the study. All tests and questionnaires were administered by investigators in a face-to-face interview.

\section{Population}

Inclusion criteria were ambulatory patients aged $\geq 40$ years with a diagnosis of COPD in the clinical records. Each patient had to present a stable condition with no worsening of symptoms over a 3-month period prior to inclusion in the study. Participants were excluded if they had been diagnosed with asthma or a chronic respiratory disease other than COPD, or if diagnosed with dementia or serious mental illness which would prevent the understanding of the questionnaires. The study protocol was approved by the
Ethics Committee of the Hospital Clinic in Barcelona on 10 September 2009 (registration number 2009/5252). All patients had to provide written informed consent to participate in the study.

\section{Measurements}

The variables obtained were gender, age, COPD-related symptoms, years since diagnosis, forced spirometry, body mass index, comorbidities, and the number of exacerbations and hospitalizations during the previous year, based on self-reporting by the patient and review of clinical records.

SES was based on the subject's occupation and educational level [5]. Occupation class was derived from the longest-held job during the professional life span. Categories were based on the major group classification, using the first digit of the International Standard Classification of Occupations [9]. The categories were as follows: class I, for managers and professionals (non-manual) of major groups 1 and 2; class II, for technicians and associate professionals of major group 3; class III, for other non-manual workers of major groups 4 and 5; class IV, for skilled manual workers of major groups 6 and 7; class V, for semi-skilled or unskilled manual workers of major groups 8 and 9 .

The educational level was based on the age of the subject at completion of full-time study. The education level was categorized as: high, finished secondary school and university degree; medium, finished primary school; low, uneducated and unfinished primary school.

The specific questionnaires used in the study were: (1) the Medical Research Council dyspnea scale [10]. (2) Severity of COPD was assessed by the COPD severity score (COPDSS), which includes questions that comprise 5 overall aspects of COPD severity: respiratory symptoms, systemic corticosteroid use, other COPD medication use, previous hospitalization or intubation for respiratory disease, and home oxygen use. Each item was assigned an a priori weight based on clinical aspects of the disease and its expected contribution to overall COPD severity. Missing values for medication use and other questions were defined as zero. Possible total scores range from 0 to 35 , and higher scores reflect more severe COPD [11] and have demonstrated predictive validity through their association with a greater prospective risk of exacerbations of COPD [12]. The questionnaire has been translated and validated into Spanish [13]. (3) The overall burden of comorbid diseases was assessed by the Charlson comorbidity score [14]. (4) The evaluation of patient HRQoL was performed by the Spanish version of the self-administered EuroQol 5-dimension questionnaire (EQ-5D) $[15,16]$, which is a generic questionnaire used to assess HRQoL in a variety of chronic diseases including COPD. The EQ-5D is comprised of a descriptive system and a visual analogue scale (VAS) which asks the respondent to consider and rate his/her health 'today'. The VAS score is anchored at 100 (best imaginable health) and 0 (worst imaginable health). The descriptive system enables the respondent to classify his/her health according to 3 levels in 5 dimensions: mobility, self-care, usual activities, pain/discomfort, and anxiety/depression. The data may be used to represent a profile of health status or may be converted into a single summary index (EQ-5D index) by applying scores from a valuation set [16]. Higher scores of the EQ-5D represent a more favorable health status. The specific respiratory HRQoL was measured by the Airways Questionnaire 20 (AQ20), developed to measure and quantify disturbances in the HRQoL of patients with asthma or COPD. The AQ20 has 20 items with yes/no responses 
and should take 2 min to complete and score. The AQ20 scores range from 0 to 20 , with a score of 0 indicating no impairment [17]; the questionnaire has recently been translated and validated into Spanish [18].

\section{Statistical Analyses}

Values are presented as the mean and standard deviation for quantitative data and as percentages for qualitative data. Mean values and standard deviations were obtained for all the variables that followed a normal distribution. The unpaired Student t test was used for comparison of independent data that followed a normal distribution. Non-normally distributed variables were compared by means of the Wilcoxon test. The Student t test for repeated measurements was used for paired data, if they followed a normal distribution. Otherwise, the Wilcoxon rank sum test was applied. The $\chi^{2}$ test was used for the comparison of categorical variables. Mean scores of the quality of life questionnaires were adjusted by age and severity (measured by the COPDSS), and adjustment was performed by using LS means in a GLM model.

The analysis was carried out using SAS software (SAS Institute, Cary, S.C., USA) version 9.1 for Windows. All tests were twotailed and the level of significance was set at 0.05 .

\section{Results}

\section{Characteristics of COPD Patients}

A total of 1,432 practicing physicians provided data from 4,891 patients; however, after checking the selection criteria, 317 (6.5\%) patients were excluded because they did not fulfill all the inclusion criteria. The remaining 4,574 patients constituted our study population.

The characteristics of the patients included in the study are described in table 1 . Most of the subjects were male (83.3\%) and had given up smoking (64.1\%). The mean age was 67.1 years (SD 10). The mean duration of COPD was 10.1 years (7.2), and the patients reported a mean of 2.4 exacerbations (2.3) and 0.9 hospital admissions (1.7) due to COPD during the last 12 months. Regarding the severity of COPD, the mean COPDSS was 10.6 (5.4) and spirometry data were obtained from 2,994 patients $(65.4 \%$ of the total) with a mean forced expiratory volume in $1 \mathrm{~s}$ (\% predicted) of $43.4 \%$ (21.9). The characteristics of the patients with and without spirometric results are presented in table 1 . No significant differences were observed in gender distribution, mean age, body mass index and severity of the disease measured by the COPDSS between groups, and therefore, both subgroups were considered to represent patients with COPD.

\section{SES and Educational Level}

Only $17.8 \%$ of the subjects belonged to occupational class I (managers and professionals), with the highest number of patients belonging to classes IV (24.8\%) and V (semi-skilled or unskilled manual workers, 31.8\%). Regarding education, up to $43 \%$ of subjects had a low educational level and only $25.4 \%$ had a high level. There were slightly more individuals in occupational class $\mathrm{V}$ among those without spirometry ( 35.0 vs. $30.3 \%$ ), but there were no significant differences in the distribution of educational levels (table 1).

\section{Patient Characteristics according to Socioeconomic and Educational Level}

Patients with a low education were significantly older, with a higher proportion of never smokers and with more comorbidities. They also had more severe COPD according to the score of the COPSS questionnaire (11.6 units of patients with low versus 10.2 and 9.3 units of those with medium and high educational levels, respectively; $\mathrm{p}<$ 0.001 ), but interestingly, no differences were observed in the degree of impairment in forced expiratory volume in $1 \mathrm{~s}$ (\% predicted). Patients with higher educational levels were diagnosed at an earlier age (table 2).

There were also differences according to occupational class, with those in the more skilled classes having an earlier diagnosis, younger age and milder disease measured by the COPDSS (table 2).

\section{Relationship between Socioeconomic and Educational Level and HRQoL}

Patients showed a moderate to severe impairment in their HRQoL, both generic and specific. The mean score of the EQ-5D index was 0.69 (SD 0.29) and of VAS 59.03 (17), and the mean AQ20 score was 9.39 (4.7).

There was a gradient of impairment in HRQoL according to educational level with significantly worse scores for the EQ-5D index and for VAS and AQ20 for medium and low educational levels compared with high education ( $\mathrm{p}<0.001)$ (table 3). Similarly, specific HRQoL was also significantly impaired in more unskilled workers with a gradient of the AQ20 from 8.6 units (SD 4.8) in class I to 10.1 units $(4.6)$ in class $\mathrm{V}(\mathrm{p}<0.001)$ and from 0.75 units in class I to 0.63 units in class V for the EQ-5D index, and from 62.9 units in class I to 55.6 units in class $\mathrm{V}$ for the EQ-5D VAS.

Since there were significant differences in age and severity of the disease between the different socioeconomic and educational classes, we evaluated the HRQoL adjusting for age and also for age and COPSS. When controlling for both variables, the differences in impairment in HRQoL were reduced but still remained significant (table 3). 
Table 1. Characteristics of the patients included in the study

\begin{tabular}{|c|c|c|c|c|}
\hline Individual variable & $\begin{array}{l}\text { Total population } \\
(\mathrm{n}=4,574)\end{array}$ & $\begin{array}{l}\text { With spirometry } \\
(\mathrm{n}=3,099)\end{array}$ & $\begin{array}{l}\text { No spirometry } \\
(\mathrm{n}=1,475)\end{array}$ & $\mathrm{p}$ value \\
\hline Males, $\mathrm{n}$ & $3,802(83.7 \%)$ & $2,600(84.4 \%)$ & $1,202(82.2 \%)$ & 0.054 \\
\hline Age, years & $67.06(10.04)$ & $67.25(10.03)$ & $66.65(10.06)$ & 0.061 \\
\hline Age at diagnosis, years & $57.24(10.3)$ & $57.78(10.28)$ & $56.05(10.23)$ & $<0.0001$ \\
\hline Body mass index & $28.02(4.32)$ & $27.97(4.43)$ & $28.13(4.06)$ & 0.27 \\
\hline \multicolumn{5}{|l|}{ Smoking status, $\mathrm{n}$} \\
\hline Never smoker & $442(9.7 \%)$ & $263(8.5 \%)$ & $179(12.2 \%)$ & \multirow[t]{3}{*}{$<0.0001$} \\
\hline Former smoker & $2,919(64.1 \%)$ & $2,059(66.8 \%)$ & $860(58.6 \%)$ & \\
\hline Current smoker & $1,190(26.2 \%)$ & $762(24.7 \%)$ & $428(29.2 \%)$ & \\
\hline \multicolumn{5}{|l|}{ Pack-years (former and } \\
\hline current smokers) & $44.31(27.56)$ & $46.12(28.52)$ & $39.94(24.57)$ & $<0.0001$ \\
\hline Charlson comorbidity index & $1.80(1.47)$ & $1.73(1.42)$ & $1.95(1.56)$ & $<0.0001$ \\
\hline \multicolumn{5}{|l|}{ Spirometry } \\
\hline FVC, 1 & $2.67(0.87)$ & $2.67(0.87)$ & & \\
\hline FVC, \% & $71.7(34)$ & $71.7(34)$ & & \\
\hline $\mathrm{FEV}_{1}, 1$ & $1.62(0.74)$ & $1.62(0.74)$ & & \\
\hline $\mathrm{FEV}_{1}, \%$ & $43.4(21.9)$ & $43.4(21.9)$ & & \\
\hline $\mathrm{FEV}_{1} / \mathrm{FVC}$ & $59(22)$ & $59(22)$ & & \\
\hline \multicolumn{5}{|c|}{ SES measures, $\mathrm{n}$} \\
\hline \multicolumn{5}{|c|}{ Occupational class $(\mathrm{n}=4,292)$} \\
\hline I & $765(17.82 \%)$ & $538(18.58 \%)$ & $227(16.26 \%)$ & \multirow[t]{5}{*}{0.003} \\
\hline II & $556(12.95 \%)$ & $375(12.95 \%)$ & $181(12.97 \%)$ & \\
\hline III & $538(12.53 \%)$ & $351(12.12 \%)$ & $187(13.40 \%)$ & \\
\hline IV & $1,065(24.81 \%)$ & $753(26.00 \%)$ & $312(22.35 \%)$ & \\
\hline $\mathrm{V}$ & $1,368(31.87 \%)$ & $879(30.35 \%)$ & $489(35.03 \%)$ & \\
\hline \multicolumn{5}{|c|}{ Educational level $(\mathrm{n}=4,556)$} \\
\hline High & $1,159(25.44 \%)$ & $786(25.44 \%)$ & $373(25.44 \%)$ & \multirow[t]{3}{*}{0.052} \\
\hline Medium & $1,435(31.50 \%)$ & $1,006(32.56 \%)$ & $429(29.26 \%)$ & \\
\hline Low & $1,962(43.06 \%)$ & $1,298(42.01 \%)$ & $664(45.29 \%)$ & \\
\hline \multicolumn{5}{|l|}{ Severity of COPD and HRQoL } \\
\hline COPDSS & $10.6(5.4)$ & $10.56(5.44)$ & $10.64(5.23)$ & 0.62 \\
\hline EQ-5D index & $0.69(0.29)$ & $0.70(0.29)$ & $0.67(0.30)$ & 0.0004 \\
\hline EQ-5D VAS & $59.3(16.5)$ & $59.23(16.77)$ & $59.53(15.95)$ & 0.62 \\
\hline AQ20 & $9.4(4.7)$ & $9.06(4.67)$ & $10.08(4.67)$ & $<0.0001$ \\
\hline
\end{tabular}

Figures in parentheses are SDs unless otherwise indicated. FVC = Forced vital capacity; FEV F $_{1}$ forced expiratory volume in $1 \mathrm{~s}$.

\section{Discussion}

This large and unselected sample of patients with COPD from primary and secondary care had a significant impairment of HRQoL, demonstrated by the scores obtained in both generic and specific HRQoL questionnaires. Patients with a lower educational level and belonging to the unskilled professional groups had a poorer HRQoL compared with the more socioeconomically advantaged individuals. The differences remained significant after controlling for age and severity of the disease, indicating that factors related to SES have an impact on the clinical manifestations, as well as on the perception and/or control of the disease by the patients.

It is well known that advanced age and severity of COPD are related to impairment of HRQoL [19, 20]. Therefore, in order to investigate the impact of other factors on HRQoL, both variables must be controlled. We used the COPDSS to control for severity. The COPDSS is a valid survey-based measure of disease-specific severity, both in terms of concurrent and predictive validity [1113]. A major advantage of this score is that it does not require lung function or other physical measurements, facilitating its use in large-scale epidemiologic studies in 
Table 2. Characteristics of the patients with COPD and severity of the disease according to SES and educational level

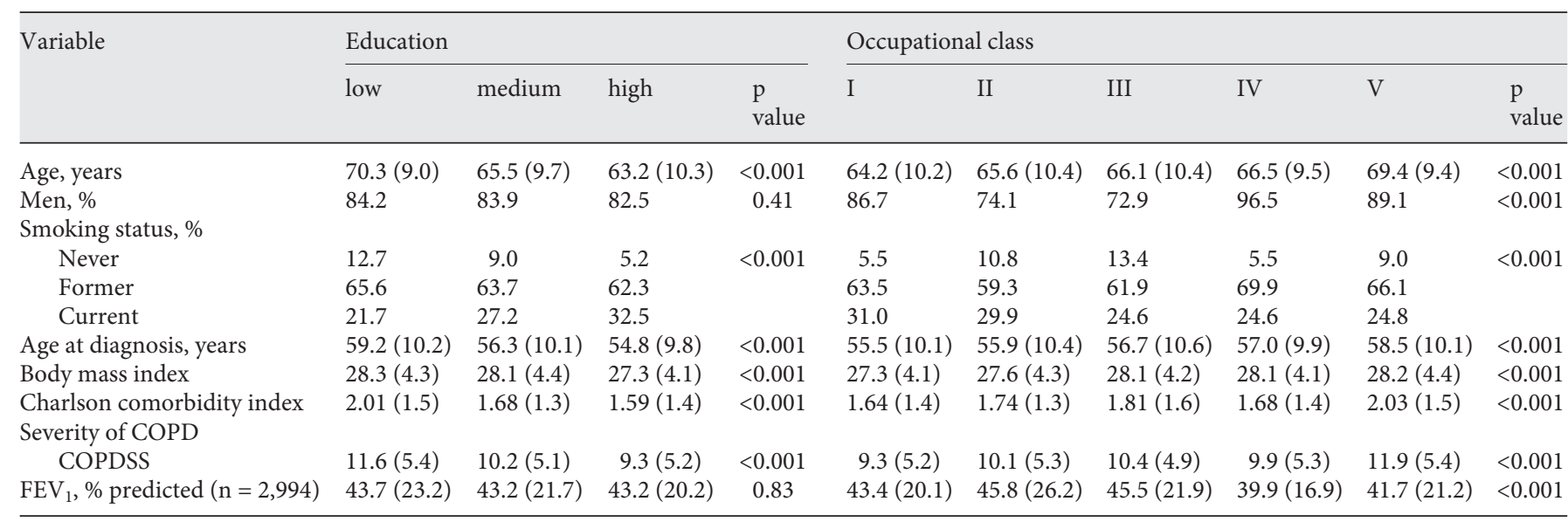

Figures in parentheses are SDs. $\mathrm{FEV}_{1}=$ Forced expiratory volume in $1 \mathrm{~s}$.

Table 3. Scores of EQ-5D index, VAS and AQ20, unadjusted and adjusted by age and severity of COPD

\begin{tabular}{|c|c|c|c|c|c|c|c|c|c|}
\hline & \multicolumn{3}{|c|}{ EQ-5D index } & \multicolumn{3}{|c|}{ EQ-5D VAS } & \multicolumn{3}{|l|}{ AQ20 } \\
\hline & $\begin{array}{l}\text { un- } \\
\text { adjusted }\end{array}$ & $\begin{array}{l}\text { age } \\
\text { adjusted }\end{array}$ & $\begin{array}{l}\text { age and } \\
\text { severity } \\
\text { adjusted }\end{array}$ & $\begin{array}{l}\text { un- } \\
\text { adjusted }\end{array}$ & $\begin{array}{l}\text { age } \\
\text { adjusted }\end{array}$ & $\begin{array}{l}\text { age and } \\
\text { severity } \\
\text { adjusted }\end{array}$ & $\begin{array}{l}\text { un- } \\
\text { adjusted }\end{array}$ & $\begin{array}{l}\text { age } \\
\text { adjusted }\end{array}$ & $\begin{array}{l}\text { age and } \\
\text { severity } \\
\text { adjusted }\end{array}$ \\
\hline \multicolumn{10}{|l|}{ SES } \\
\hline Low & 0.63 & 0.64 & 0.66 & 56.3 & 56.9 & 58.1 & 10.09 & 10.10 & 9.72 \\
\hline $\mathrm{p}$ value & $<0.0001$ & $<0.0001$ & $<0.0001$ & $<0.0001$ & $<0.0001$ & $<0.0001$ & $<0.0001$ & $<0.0001$ & $<0.0001$ \\
\hline \multicolumn{10}{|c|}{ Occupational } \\
\hline I & 0.75 & 0.73 & 0.70 & 62.9 & 61.9 & 60.3 & 8.61 & 8.62 & 9.10 \\
\hline II & 0.69 & 0.68 & 0.67 & 60.3 & 59.2 & 58.6 & 9.25 & 9.24 & 9.43 \\
\hline III & 0.69 & 0.68 & 0.68 & 59.8 & 59.6 & 59.5 & 9.78 & 9.77 & 9.81 \\
\hline
\end{tabular}

COPD. It is well recognized that spirometry is not widely available in primary care, and quality control may be difficult in large-scale studies [21-23] which also limits the possible application of other severity scales such as the BODE index [24]. In our series, spirometry was available in $65.4 \%$ of patients, but comparison of basic clinical and demographic characteristics between patients with and without spirometry allowed meaningful differences between both groups to be ruled out, and therefore, we could reasonably assume that the vast majority of patients were correctly classified as COPD.
Women with COPD may display a more severe impairment of HRQoL at the same level of physiological impairment [25]. However, we did not control for gender because women were equally distributed in all educational levels and were underrepresented in the total sample of participants, consistently with the epidemiology of COPD in Spain, where more than $75 \%$ of COPD cases are men $[7,8]$.

The relationship between SES and lung function, chronic bronchitis or COPD has been extensively analyzed in population-based studies that have consistently 
reported a higher incidence of chronic respiratory disease and a reduced lung function associated with a low SES [1, $3-5]$. It is interesting to observe the characteristics of COPD in low educational levels, where the proportion of never smokers and the comorbidity index were higher. This suggests that other risk factors could be important in this disadvantaged population, such as professional exposure to dusts and chemicals, a higher incidence of tuberculosis [26] and unhealthy behaviors. In fact, there is an association between low SES and a higher risk of death, explained in great part by the higher prevalence of an unhealthy diet, low physical activity and higher alcohol consumption [27]. Specifically regarding lung health, a strong social gradient in respiratory mortality and COPD has been described that is independent of smoking and is stronger in males [6]. Similarly, low SES was associated with increased lung function decline in males in a cohort study in the general population [28], and having no educational qualifications and living in a deprived area independently predicted significantly lower lung function, even after controlling for smoking habit [29]. The results of a population-based epidemiological study in Spain showed that individuals with less than primary school education had a $60 \%$ increased risk of having COPD [7] and had a $46 \%$ increased risk of dying by COPD in the lowest quartile compared with the highest quartile of SES [30]. Our results indicate that more skilled individuals with COPD were younger, had an earlier COPD diagnosis and milder disease compared with unskilled subjects, despite having a health care system that is universally available and free of cost. This is consistent with a previous study that observed that individuals with COPD detected in an epidemiological survey with $<12$ years of education had a 77\% significantly increased likelihood of not having been previously diagnosed compared with those with $>12$ years of education [8]. Similarly, it has been described that unemployed subjects, besides having a higher risk of chronic bronchitis and bronchitis-type symptoms, consistently reported less frequent contact with specialized practitioners, and therefore, are less likely to undergo spirometry [31]. In a previous international survey, COPD patients belonging to high SES were significantly younger and had a milder impairment in lung function. Additionally, they were more likely to receive long-acting respiratory drugs and to be enrolled in rehabilitation programs [32]. These findings underscore that health education and awareness play a key role in the prevention of COPD and its development.

All the evidence indicates that even in countries where the access to health care services is universal and free, socioeconomic disadvantage is an independent risk factor for COPD, is independently associated with a worse HRQoL and represents a barrier for early detection and better management of the disease.

\section{Acknowledgements}

The INSEPOC study has been funded by an unrestricted grant from Laboratorios Dr. Esteve, S.A., Spain.

\section{Financial Disclosure and Conflicts of Interest}

The authors declare that they have no competing interests.

\section{References}

1 Prescott E, Vestbo J: Socioeconomic status and chronic obstructive pulmonary disease. Thorax 1999;54:737-741.

-2 Basagaña X, Sunyer J, Kogevinas M, Zock JP, Duran-Tauleria E, Jarvis D, Burney P, Anto JM: Socioeconomic status and asthma prevalence in young adults: the European Community Respiratory Health Survey. Am J Epidemiol 2004;160:178-188.

-3 Prescott E, Lange P, Vestbo J, the Copenhagen City Heart Study Group: Socioeconomic status, lung function and admission to hospital for COPD: results from the Copenhagen City Heart Study. Eur Respir J 1999;13: 1109-1114.

4 Hegewald MJ, Crapo RO: Socioeconomic status and lung function. Chest 2007;132: 1608-1614.
5 Ellison-Loschmann L, Sunyer J, Plana E, Pearce N, Zock JP, Jarvis D, Janson C, Antó JM, Kogevinas M: Socioeconomic status, asthma and chronic bronchitis in a large community-based study. Eur Respir J 2007; 29:897-905.

6 Prescott E, Godtfredsen N, Vestbo J, Osler M: Social position and mortality from respiratory diseases in males and females. Eur Respir J 2003;21:821-826.

-7 Miravitlles M, Soriano JB, García-Río F, Muñoz L, Duran-Taulería E, Sánchez G, Sobradillo V, Ancochea J: Prevalence of COPD in Spain: impact of undiagnosed COPD on quality of life and daily life activities. Thorax 2009;64:863-868.
8 Sobradillo V, Miravitlles M, Gabriel R, Jiménez-Ruiz CA, Villasante C, Masa JF, Viejo JL, Fernández-Fau L: Geographical variations in prevalence and underdiagnosis of COPD. Results of the IBERPOC multicentre epidemiological study. Chest 2000;118:981-989.

9 International Labour Office: International Standard Classification of Occupations: ISCO-88. Geneva, International Labour Office, 1990.

10 Bestall JC, Paul EA, Garrod R, Garnham R, Jones PW, Wedzicha JA: Usefulness of the Medical Research Council (MRC) dyspnoea scale as a measure of disability in patients with chronic obstructive pulmonary disease. Thorax 1999;54:581-586. 
11 Eisner MD, Trupin L, Katz PP, Yelin EH, Earnest G, Balmes J, Blanc PD: Development and validation of a survey-based COPD Severity Score. Chest 2005;127:1890-1897.

- 12 Eisner MD, Omachi TA, Katz PP, Yelin EH, Iribarren C, Blanc PD: Measurement of COPD severity using a survey-based score: validation in a clinically and physiologically characterized cohort. Chest 2010;137:846851.

13 Miravitlles M, Llor C, de Castellar R, Izquierdo I, Baró E, Donado E: Validation of the COPD severity score for its use in primary care. The NEREA study. Eur Respir J 2009; 33:519-527.

$\checkmark 14$ Charlson ME, Szatrowski TP, Peterson J, Gold J: Validation of a combined comorbidity index. J Clin Epidemiol 1994;47:1245 1251.

-15 The EuroQol Group: EuroQol - a new facility for measurement of health-related quality of life. Health Policy 1990;16:199-208.

16 Badia X, Roset M, Montserrat S, Herdman M, Segura A: The Spanish version of EuroQol: a description and its applications. European Quality of Life scale. Med Clin (Barc) 1999;112(suppl 1):79-85.

- 17 Hajiro T, Nishimura K, Jones PW, Tsukino M, Ikeda A, Koyama H, Izumi T: A novel, short, and simple questionnaire to measure health-related quality of life in patients with chronic obstructive pulmonary disease. Am J Respir Crit Care Med 1999;159:1874-1878.

18 Blanco-Aparicio M, Vázquez-Rodríguez I, Verea-Hernando $\mathrm{H}$ : Cultural adaptation into Spanish of the Airways Questionnaire 20, a short health-related quality of life questionnaire for the clinical evaluation of asthma and chronic obstructive pulmonary disease. Arch Bronconeumol 2009;45:24-29.
9 Ferrer M, Alonso J, Morera J, Marrades RM, Khalaf A, Aguar C, Plaza V, Prieto L, Antó JM: Chronic obstructive pulmonary disease stage and health-related quality of life. Ann Intern Med 1997;127:1072-1079.

20 Miravitlles M, Ferrer M, Pont A, Viejo JL, Masa JF, Gabriel R, Jiménez-Ruiz CA, Villasante C, Fernandez-Fau L, Sobradillo V: Characteristics of a population of COPD patients identified from a population-based study. Focus on previous diagnosis and never smokers. Respir Med 2005;99:985-995.

21 Miravitlles M, de la Roza C, Naberan K, Lamban M, Gobartt E, Martín A: Use of spirometry and patterns of prescribing in COPD in primary care. Respir Med 2007; 101:1753-1760.

22 Derom E, van Weel C, Liistro G, Buffels J, Schermer T, Lammers E, Wouters E, Decramer M: Primary care spirometry. Eur Respir J 2008;31:197-203.

-23 Naberan K, de la Roza C, Lamban M, Gobartt E, Martín A, Miravitlles M: Use of spirometry in the diagnosis and treatment of chronic obstructive pulmonary disease in primary care. Arch Bronconeumol 2006;42:638-644

24 Celli BR, Cote C, Marin JM, Casanova C, Montes M, Méndez RA, Pinto V, Cabral HH The body mass index, airflow obstruction, dyspnea, and exercise capacity index in chronic obstructive pulmonary disease. $\mathrm{N}$ Engl J Med 2004;350:1005-1012.

25 Carrasco-Garrido P, de Miguel-Díez J, Rejas-Gutierrez J, Martín-Centeno A, GobarttVázquez E, Hernández-Barrera V, Gil de Miguel A, Jiménez-García R: Characteristics of chronic obstructive pulmonary disease in Spain from a gender perspective. BMC Pulm Med 2009;9:2.
26 Menezes AMB, Hallal PC, Perez-Padilla R, Jardim JRB, Muiño A, Lopez MV, Valdivia G, Montes de Oca M, Talamo C, Pertuze J, Victoria CG: Tuberculosis and airflow obstruction: evidence from the PLATINO study in Latin America. Eur Respir J 2007;30: 1180-1185.

27 Stringhini S, Sabia S, Shipley M, Brunner E, Nabi H, Kivimaki M, Singh-Manoux A: Association of socio-economic position with health behaviours and mortality. JAMA 2010;303:1159-1166.

28 Johannessen A, Eagan TML, Omenaas ER, Bakke PS, Gulsvik A: Socioeconomic risk factors for lung function decline in a general population. Eur Respir J 2010;36:480-487.

29 Shohaimi S, Welch A, Bingham S, Luben R, Day N, Wareham N, Khaw KT: Area deprivation predicts lung function independently of education and social class. Eur Respir J 2004; 24:157-161.

30 Regidor E, Domínguez V, Calle ME, Navarro $\mathrm{P}$ : Socioeconomic circumstances and premature mortality from chronic diseases. Med Clin (Barc) 2003;120:201-206.

31 Kogevinas M, Antó JM, Tobias A, Alonso J, Soriano J, Almar E, Muniozguren N, Payo F, Pererira A, Sunyer J, for the Spanish Group of the European Community Respiratory Health Survey: Respiratory symptoms, lung function and use of health services among unemployed young adults in Spain. Eur Respir J 1998;11:1363-1368.

-32 Miravitlles M, Murio C, Tirado-Conde G, Levy G, Muellerova H, Soriano JB, RamirezVenegas A, Ko FWS, Canelos-Estrella B, Giugno E, Bergna M, Chérrez I, Anzueto A: Geographic differences in clinical characteristics and management of COPD: the EPOCA study. Int J Chron Obstruc Pulmon Dis 2008;3:803-814. 